Gut, 1968, 9, 139-143

\title{
Effect of irritant purgatives on the myenteric plexus in man and the mouse
}

\author{
BARBARA SMITH \\ From the Department of Pathology, St. Bartholomew's Hospital, London
}

Purgatives are some of the most widely used of all drugs, particularly preparations of cascara sagrada and senna, the anthraquinone cathartics. In spite of this comparatively little work has been done on their mode of action. Straub and Triendl (1937) showed that senna would act when given parenterally but only after a latent period of up to eight hours. The authors suggested that during this period it is converted into the active anthraquinone and excreted into the large gut. They also showed that it is active when given by mouth to an animal with a divided intestine. Grabfield (1937) perfused isolated coils of small and large intestine with 1 in 10,000 cascara showing that it produced an increase in peristalsis in the latter but not in the former. Okada (1940) suggested that the Escherischia coli converted senna to an oxyanthraquinone which acted on Auerbach's plexus. He considered that the resulting pressure changes which he recorded were similar to normal peristalsis. In view of the theory then current that the anthraquinone purgatives acted by irritating the mucosa, producing a reflex muscular contraction, Douthwaite and Goulding (1957) gave sennakot to mice. They produced purgation but were unable to show any evidence of inflammation in the gut. They commented that the mode of action of the drug was unknown. Thus the rather scanty evidence suggests that the drug could have a direct effect on myenteric neurones. Corroborative evidence for this theory comes from patients who have been taking purgatives for many years and who become resistant to their effect. It is almost impossible to wear out a physiological reflex in the presence of normal neurones so possibly the ganglion cell itself is damaged. In this work the pathology of the plexus in a patient who had been taking purgatives for over 40 years will be described and also the effect on this structure of giving senna to mice.

\section{CLINICAL HISTORY}

The details of this patient's history were presented more fully to the Royal Society of Medicine by Dr. Avery
Jones (1967). The patient began to have increasing constipation from the age of 13 and frequently took aperients. She was subject to attacks of a feeling of distension and abdominal pain. She had a loop of sigmoid removed at the age of 50 without relief and continued to take laxatives. At the age of 57 she was passing three or four fluid stools a day. Colectomy was done at the age of 57 and resulted in relief of symptoms.

\section{PATHOLOGY}

I am indebted to Dr. Basil Morson for the following macroscopic description of the specimen.

'It consists of the terminal $5.0 \mathrm{~cm}$ of ileum with the colon from the caecum to the rectum. The ileocaecal valve is stenosed and incompetent. The colon shows loss of interhaustral folds throughout. The mucous membrane is dark brown and has the texture of snake skin, showing an appearance of reticulation with a network of fine white intercommunicating lines. The submucosa is filled with adipose tissue and at one point in the transverse colon this is thick enough to produce a polypoid tumour $2.0 \mathrm{~cm}$ in diameter. The muscle layers show obvious atrophy.'

The plexus was demonstrated by silver impregnation of sections cut parallel to the gut wall as described previously (Smith, 1967). The abnormal features were a vast increase in the number of Schwann cells which filled the plexus region, and a considerable reduction in the number of neurones and axons. The neurones which remained were poorly argentophilic with irregular, ill-defined margins and only one or two swollen processes. The axons ran singly and not in bundles, they were irregular in calibre, and a few were random in direction, apparently regenerating fibres (Figs. 1, 2, and 3).

\section{RESULTS}

In the mouse the intestinal contents are dehydrated and formed into pellets in the proximal colon and passed distally. There is no significant storage of faeces in these animals and they are excreted quite quickly. Normally the distal colon does not contain more than two or occasionally three well-spaced pellets. If a purgative is given and the animal is killed about eight hours later, the distal colon is empty. If a large dose is given, the colon may be 


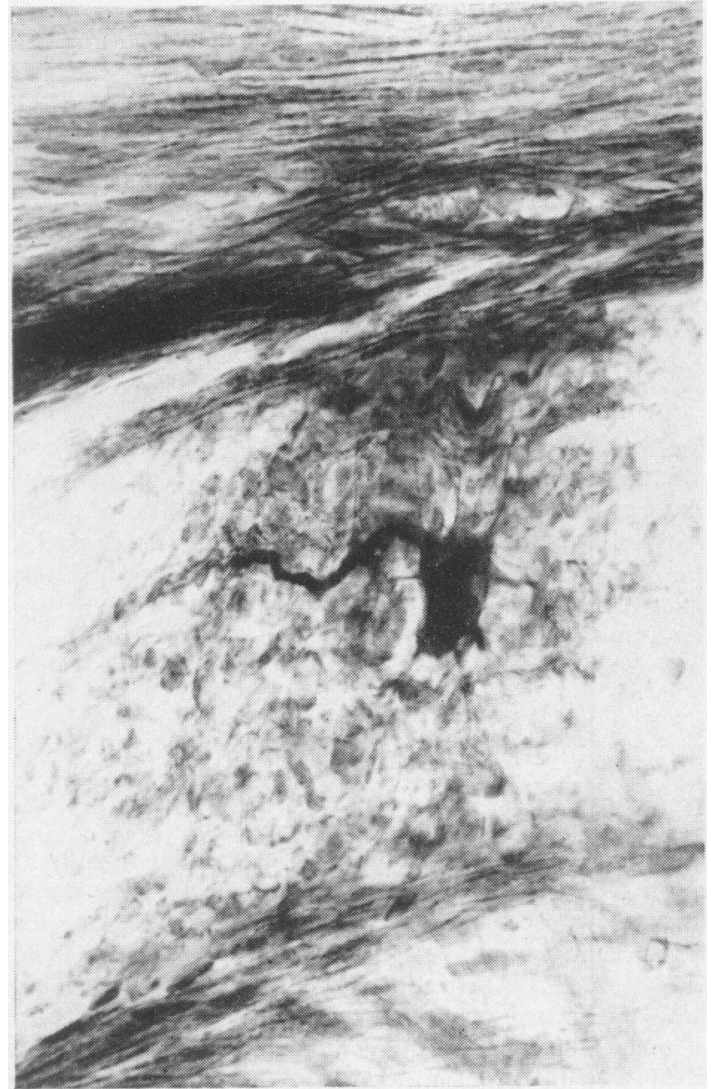

FIG. 1.

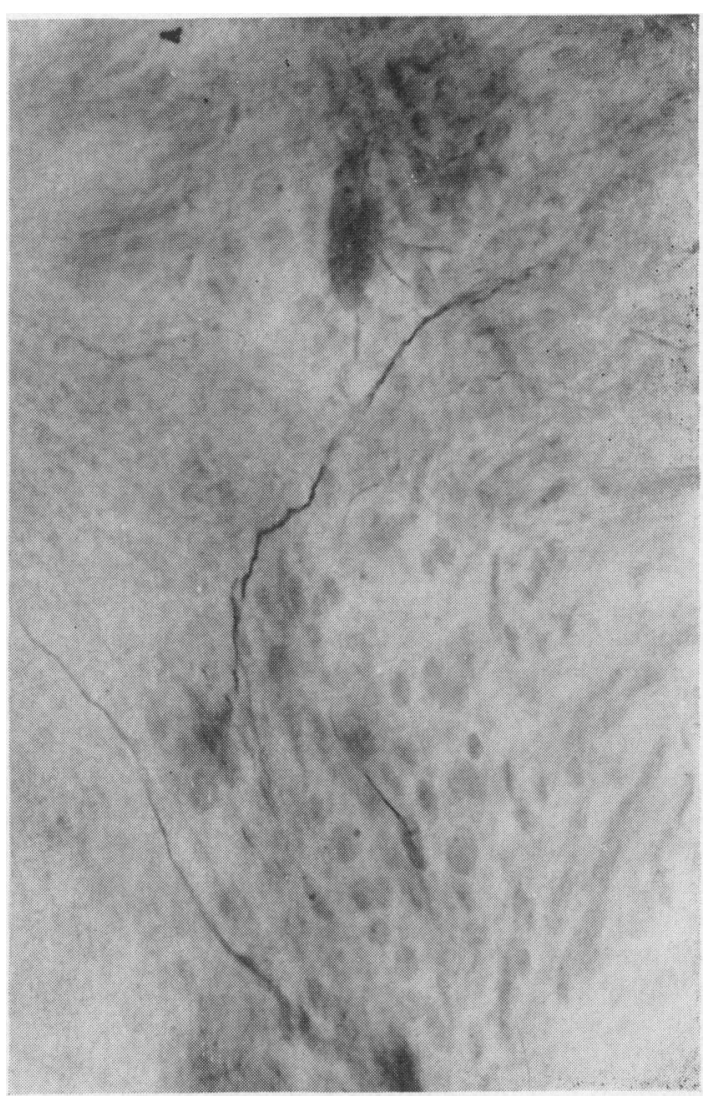

FIG. 2.

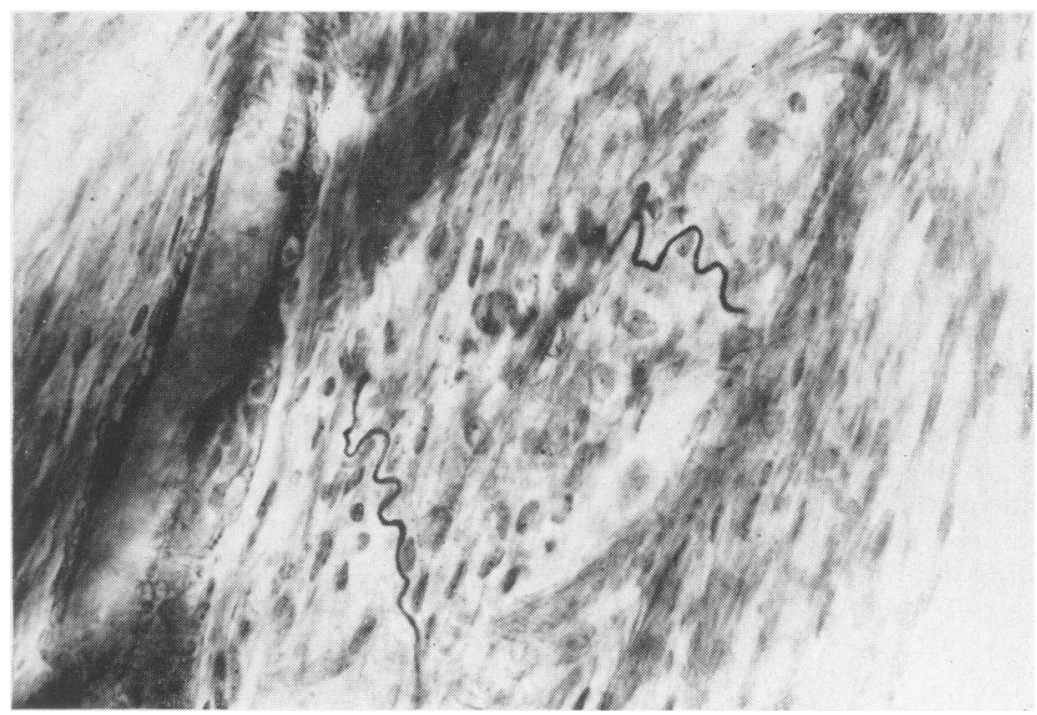

FIG. 1. Cathartic colon. A single neurone remaining in a ganglion with a grossly thickened axon $(\times 300)$.

FIG. 2. Cathartic colon. A poorly staining neurone and two or three axons remaining in a ganglion. Schwann cell nuclei can be seen in the background $(\times 300)$.

FIG. 3. Cathartic colon. Two tortuous axons which are probably regenerating. There are no neuronal nuclei in this ganglion $(\times 300)$.

FIG. 3. 


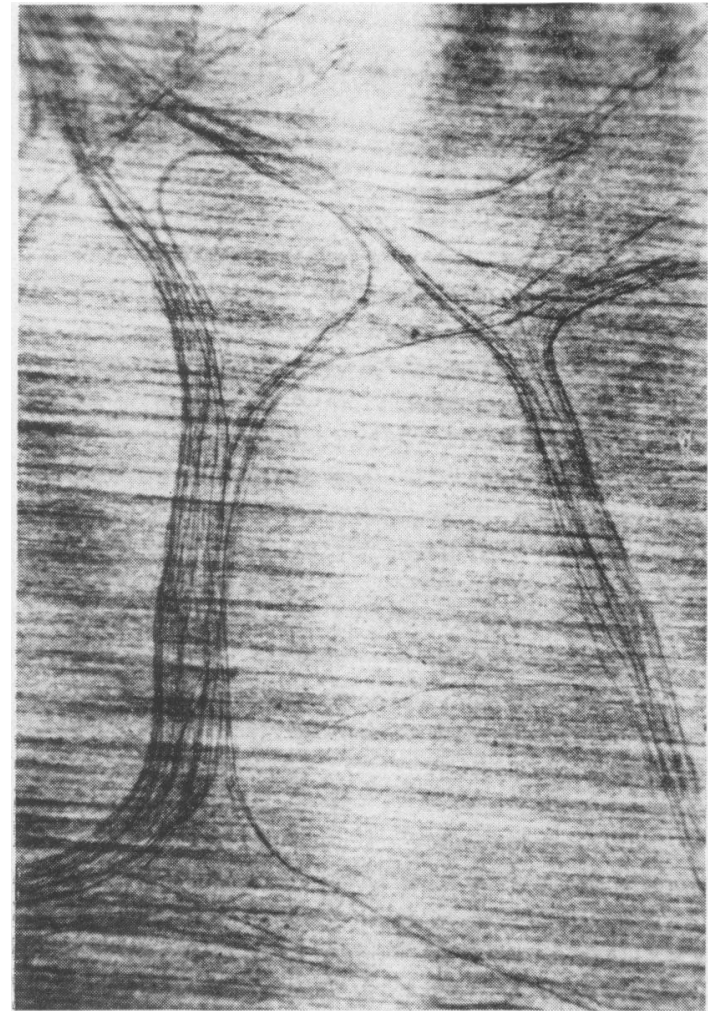

FIG. 4.

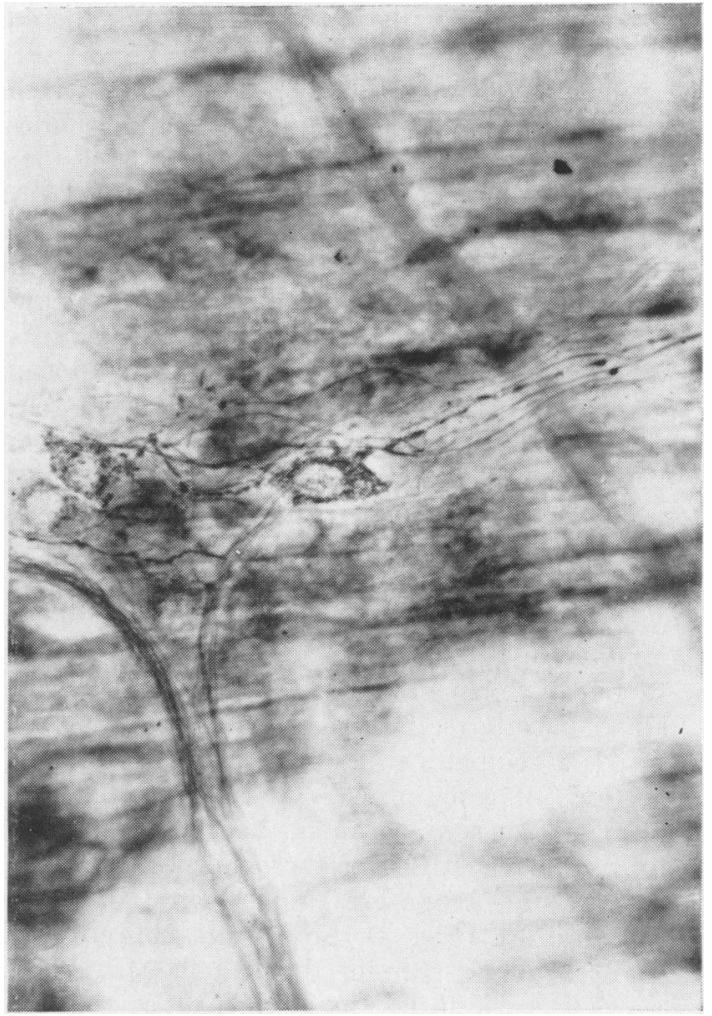

FIG. 5 .

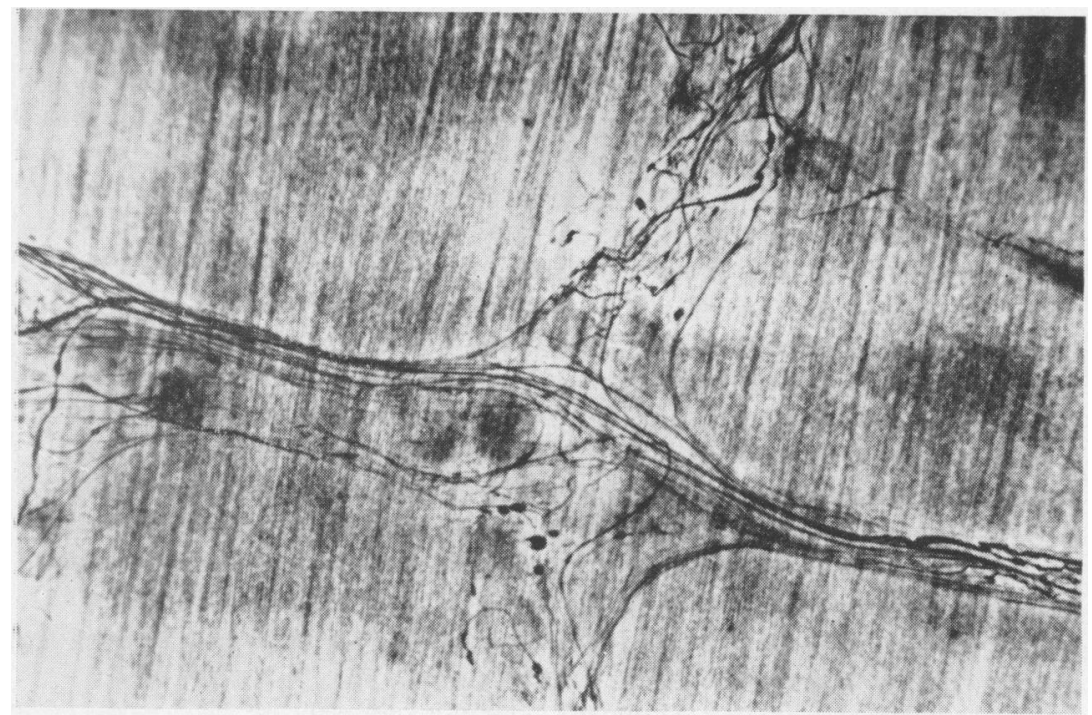

FIG. 4. Normal mouse myenteric plexus. The axons are fine, straight and regular. Neurones are present but do not stain $(\times 300)$.

FIG. 5. The myenteric plexus of a mouse given syrup of senna, 1 in 20 , by mouth for five weeks. Neurones are argentophil. The axons show beading and fragmentation $(\times 300)$.

FIG. 6. The myenteric plexus of a mouse given syrup of senna, 1 in 100, intraperitoneally for 10 daily doses. There are a number of dendritic enlargements which are probably swollen boutons terminaux $(\times 300)$.

FIS. 6. 
empty as far back as the ileocaecal valve. If there is impaired peristalsis, the pellets will be excreted more slowly and the distal colon will be full. In the animals given senna for more than a fortnight, intraperitoneally, the distal colon always contained more than three pellets; often it was tightly packed with five or six. Of the animals taking senna by mouth, 22 out of 30 showed similar changes. In nearly all the animals the faeces were larger and softer than normal due to impaired water absorption.

When the colon is removed from the normal mouse it immediately undergoes segmental contraction and the lumen is almost obliterated. Experience with other neurotoxic drugs (Smith, 1968) suggest that when the plexus is damaged this fails to occur and the gut remains flabby and open. In the animals given the senna intraperitoneally, in 14 out of 20 contraction was impaired in response to trauma, and of those taking the drug by mouth, in 20 out of thirty.

Histologically the normal myenteric plexus of the mouse is a geometrical pattern of nerve trunks with collections of ganglion cells at their junctions and to some extent running along their length. When it is impregnated with silver the neurones are usually unstained, the axons are smooth, straight and of regular calibre (Fig. 4). Sometimes collections of dendrites can be seen in the ganglia. In animals given neurotoxic drugs the abnormalities consist of swelling and argentophilia of neurones, axonal fragmentation, retraction balls, and dendritic masses which are probably swollen boutons terminaux (Hoff and Hoff, 1934) and neurofibrillary tangles probably representing hypertrophy of the autonomic ground plexus (Smith, 1968). In the animals given syrup of senna by mouth, there was little change for about a month. After this there was slight neuronal argentophilia with swelling of axons and some neuro-fibrillary tangles. After four months, there was evidence of axonal fragmentation and dendritic swelling (Fig. 5). In the animals given the drug intraperitoneally, there was considerable damage evident after a fortnight. There was a little argentophilia of neurones but a more striking feature was the presence of large numbers of axon swellings and boutons terminaux (Fig. 6).

\section{DISCUSSION}

The histological changes produced in the myenteric plexus in animals by neurotoxic drugs are somewhat variable. Some substances, such as the vinca alkaloids, seem to produce gross argentophilia with axonal fragmentation; others, such as mepacrine, produce much less argentophilia but large numbers of axonal and dendritic swellings
(Smith, 1968). The appearances in the animals given senna, particularly by the parenteral route, which gives a higher dose, are very similar to those produced by mepacrine. It is interesting that mepacrine, which is fluorescent, can be shown to have a specific affinity for neurones rendering them visible when viewed by ultraviolet light. The acridines and the anthraquinones are chemically allied and it is possible that they may affect neurones in a similar way. The rather specific affinity of senna for the large intestine may be due to the fact that it is secreted into it, and very small doses will affect the neurones in the gut wall because it is concentrated there. It has thus been shown that senna has a histologically damaging effect on myenteric neurones when given to mice. It is therefore suggested that the drug has an affinity for these cells and acts in the pharmacological situation by stimulating them.

The patient who had been taking purgatives for many years also had marked damage to the plexus. However, here the situation is more complicated, because she had been taking a number of different drugs and it is not really known why she started to take laxatives originally. Acquired organic disease of the plexus producing failure of gut motility does exist and such patients will inevitably take aperients. Thirty years later it is impossible to say whether the damage is entirely due to purgatives or whether there was a primary plexus lesion. Patients who have an inflammatory destruction of the plexus both in Chagas' disease and in idiopathic megacolon have a considerable hypertrophy of the smooth muscle particularly the inner coat (personal observation). This is possibly a true work hypertrophy due to increased segmentation not mediated neurologically in the absence of coordinated peristalsis. This patient had a definite atrophy of the bowel wall which is perhaps against a primary organic lesion.

In the cathartic syndrome, several fluid motions a day are passed. The myenteric plexus in the distal colon is not only responsible for peristalsis but also by active relaxation for storage. If the damage is sufficiently severe, the colon may be converted into an inert tube through which small intestinal contents pass, helped along by segmentation and a push from behind, and no storage occurs. Hence, these patients lose considerable quantities of water and potassium.

\section{SUMMARY}

Myenteric plexus damage is described in a patient with the cathartic syndrome.

Damage to the myenteric plexus was produced in mice by the administration of syrup of senna. 
It is suggested that senna acts pharmacologically by its effect on myenteric neurones, and if taken long enough may damage them.

This work was assisted by a grant from The Muscular Dystrophy Group of Great Britain. I am grateful to Mr. P. Crocker for the photographs.

\section{REFERENCES}

Douthwaite, A. H., and Goulding, R. (1957). Action of senna. Brit. med. J., 2, 1414-1415.
Grabfield, G. P. (1937). New experiments on some old cathartics. J. Pharmacol. exp. Ther., 60, 108.

Hoff, E. C., and Hoff, H. E. (1934). Spinal terminations of the projection fibres from the motor cortex of primates. Brain, 57, 454-474.

Jones, F. A. (1967). Cathartic colon. Proc. roy. Soc. Med., 60, 503-504.

Okada, T. (1940). Uber den Mechanismus der Sennaabführung. Tohoku J. exp. Med., 38, 33-44.

Schofield, G. C. (1960). Experimental studies on the innervation of the mucous membrane of the gut. Brain, 83, 490-514.

Smith, B. (1967). Myenteric plexus in Hirschsprung's disease. Gut, 8, 308-312.

- (1968). The myenteric plexus in drug-induced neuropathy. J. Neurol. Neurosurg. Psychiat., 30, 506-510.

Straub, W., and Triendl, E. (1937). Theorie der Abführwirkung der Folia Sennae und ihrer wirksamen Inhaltstoffe. NaunynSchmiedeberg's Arch. exp. Path. Pharmak., 185, 1-19. 